\title{
On bases from perturbed system of exponents in Lebesgue spaces with variable summability exponent
}

Togrul Muradov*

\section{${ }^{*}$ Correspondence:}

togrulmuradov@gmail.com Department of Non-harmonic Analysis, Institute of Mathematics and Mechanics of NAS of Azerbaijan, 9 B. Vahabzadeh Str., Baku, Azerbaijan

\begin{abstract}
In this paper the perturbed system of exponents with some asymptotics is considered. Basis properties of this system in Lebesgue spaces with variable summability exponent are investigated.
\end{abstract}

Keywords: system of exponents; perturbation; generalized Lebesgue space; variable exponent

\section{Introduction}

Consider the following system of exponents:

$$
\left\{e^{i \lambda_{n} t}\right\}_{n \in Z}
$$

where $\left\{\lambda_{n}\right\} \subset R$ is a sequence of real numbers, $Z$ is a set of integer numbers. It is the aim of this paper to investigate basis properties (basicity, completeness, and minimality) of the system (1) in Lebesgue space $L_{p_{t}}$ with variable summability index $p(t)$, when $\left\{\lambda_{n}\right\}$ has the asymptotics

$$
\lambda_{n}=n-\alpha \operatorname{sign} n+O\left(|n|^{-\beta}\right), \quad n \rightarrow \infty,
$$

where $\alpha, \beta \in R$ are some parameters.

Many authors have investigated the basicity properties of system of exponents of the form (1), beginning with the well-known result of Paley and Wiener [1] on Riesz basicity. Some of the results in this direction have been obtained by Young [2]. The criterion of basicity of the system (1) in $L_{p} \equiv L_{p}(-\pi, \pi), 1<p<+\infty$, when $\lambda_{n}=n-\alpha$ sign $n$, has been obtained earlier in $[3,4]$.

Recently in connection with consideration of some specific problems of mechanics and mathematical physics [5, 6], interest in the study of the various questions connected with Lebesgue $L_{p_{t}}$ and Sobolev $W_{p_{t}}^{k}$ spaces with variable summability index $p(t)$ has increased [5-9].

Many questions of the theory of operators (for example, theory of singular operators, theory of potentials and etc.) are studied in spaces $L_{p_{t}}$ [7]. These investigations have allowed one to consider questions of basicity of some system of functions (for example, the 
classical system of exponents $\left.\left\{e^{i n t}\right\}_{n \in Z}\right)$ in $L_{p_{t}}$. In [9] the basicity of system $\left\{e^{i n t}\right\}_{n \in N}$ in $L_{p_{t}}$ has been established. The special case of the system (1) is considered in [10-12], when $\lambda_{n}=n-\alpha \operatorname{sign} n, n \in Z$.

In this paper basis properties of the system (1) in $L_{p_{t}}$ spaces are investigated. Under certain conditions on the parameters $\alpha$ and $\beta$ equivalence of the basis properties (completeness, minimality, $\omega$-linearly independence, basicity) of the system (2) in $L_{p_{t}}$ are proved.

\section{Necessary notion and facts}

Let $p:[-\pi, \pi] \rightarrow[1,+\infty)$ be a Lebesgue measurable function. By $\mathrm{L}_{0}$ we denote the class of all functions measurable on $[-\pi, \pi]$ with respect to Lebesgue measure. We choose the notation

$$
I_{p}(f) \stackrel{\text { def }}{=} \int_{-\pi}^{\pi}|f(t)|^{p(t)} d t .
$$

Let $\mathrm{L} \equiv\left\{f \in \mathrm{L}_{0}: I_{p}(f)<+\infty\right\}$. Let $p^{-}=\inf \operatorname{vrai}_{[-\pi, \pi]} p(t), p^{+}=\sup \operatorname{vrai}{ }_{[-\pi, \pi]} p(t)$. For $p^{+}<$ $+\infty$, with respect to ordinary linear operations of addition of functions and multiplication by number, $\mathrm{L}$ turns into a linear space. If we define in $L_{p_{t}}$ the norm

$$
\|f\|_{p_{t}} \stackrel{\text { def }}{=} \inf \left\{\lambda>0: I_{p}\left(\frac{f}{\lambda}\right) \leq 1\right\}
$$

then $\mathrm{L}$ is a Banach space and we denote it by $L_{p_{t}}$. Denote

$$
\begin{aligned}
H^{\ln } & \stackrel{\text { def }}{=}\left\{p: p(\pi)=p(-\pi) \text { and } \exists C>0, \forall t_{1}, t_{2} \in[-\pi, \pi],\left|t_{1}-t_{2}\right| \leq \frac{1}{2}\right. \\
& \left.\Rightarrow\left|p\left(t_{1}\right)-p\left(t_{2}\right)\right| \leq \frac{C}{-\ln \left|t_{1}-t_{2}\right|}\right\} .
\end{aligned}
$$

Throughout this paper, $q(t)$ denotes the function conjugate to function $p(t)$, that is, $\frac{1}{p(t)}+$ $\frac{1}{q(t)} \equiv 1$.

We have Hölder's generalized inequality,

$$
\int_{-\pi}^{\pi}|f(t) g(t)| d t \leq C\left(p^{-} ; p^{+}\right)\|f\|_{p_{t}}\|g\|_{q t}
$$

where $C\left(p^{-} ; p^{+}\right)=1+\frac{1}{p^{-}}-\frac{1}{p^{+}}$.

For our investigation we need some basic concepts of the theory of close bases, given as follows.

We adopt the standard notation: $B$-space is a Banach space; $X^{*}$ is the conjugate to space $X ; f(x), f \in X^{*}$, and $x \in X$ means the value of functional $f$ on $x ; L[M]$ is a linear span of a set $M$. The system $\left\{x_{n}\right\}_{n \in N} \subset X$ is called $\omega$-linear independent in $B$-space $X$, if $\sum_{n=1}^{\infty} \alpha_{n} x_{n}=0$ true for $\alpha_{n}=0, \forall n \in N$.

The following lemma is true.

Lemma 1 Let $X$ be a Banach space with basis $\left\{x_{n}\right\}_{n \in N} \subset X$ and $F: X \rightarrow X$ be a Fredholm operator. Then the following properties of the system $\left\{y_{n}=F x_{n}\right\}_{n \in N}$ in $X$ are equivalent:

(1) $\left\{y_{n}\right\}_{n \in N}$ is complete; 
(2) $\left\{y_{n}\right\}_{n \in N}$ is minimal;

(3) $\left\{y_{n}\right\}_{n \in N}$ is $\omega$-linear independent;

(4) $\left\{y_{n}\right\}_{n \in N}$ is isomorphic to $\left\{x_{n}\right\}_{n \in N}$ basis.

We also need the following easily provable lemma.

Lemma 2 Let $X$ be a Banach space with basis $\left\{x_{n}\right\}_{n \in N}$ and $\left\{y_{n}\right\}_{n \in N} \subset X: \operatorname{card}\left\{n: x_{n} \neq y_{n}\right\}<$ $+\infty$. Then the expression

$$
F x=\sum_{n=1}^{\infty} x_{n}^{*}(x) y_{n}
$$

generates the Fredholm operator $F: X \rightarrow X$, where $\left\{x_{n}^{*}\right\}_{n \in N} \subset X^{*}$ is conjugate to $\left\{x_{n}\right\}_{n \in N}$ system.

The following lemma is also true.

Lemma 3 Let $\left\{x_{n}\right\}_{n \in N}$ be complete and minimal in B-space $X$ and $\left\{y_{n}\right\}_{n \in N} \subset X: \operatorname{card}\{n$ : $\left.x_{n} \neq y_{n}\right\}<+\infty$. Then the following properties of system $\left\{y_{n}\right\}_{n \in N}$ in $X$ are equivalent:

(1) $\left\{y_{n}\right\}_{n \in N}$ is complete;

(2) $\left\{y_{n}\right\}_{n \in N}$ is minimal.

These and other results are obtained in $[13,14]$.

We will use the following statement, which has a proof similar to the proof of Levinson [15].

Statement 1 Let system $\left\{e^{i \lambda_{n} t}\right\}_{n \in Z}$ be complete in $L_{p_{t}}$. If from the system we remove $n$ any functions and add instead of them $n$ other functions $e^{i \mu_{j} t}, j=1, \ldots, n$, where $\mu_{1}, \ldots, \mu_{n}$ are any, mutually different complex numbers not equal to any of numbers $\lambda_{k}$, then the new system will be complete.

We shall also need the following theorem of Krein-Milman-Rutman.

Theorem 1 (Krein-Milman-Rutman [13]) Let $X$ be a Banach space with norm $\|\cdot\|$, $\left\{x_{n}\right\}_{n \in N} \subset X$ be normed basis in $X$ (i.e. $\left\|x_{n}\right\|=1, \forall n \in N$ ) with conjugate system $\left\{x_{n}^{*}\right\}_{n \in N} \subset$ $X^{*}$, and $\left\{y_{n}\right\}_{n \in N} \subset X$ be a system satisfying the inequality

$$
\sum_{n=1}^{\infty}\left\|x_{n}-y_{n}\right\|<\gamma^{-1}
$$

where $\gamma=\sup _{n}\left\|x_{n}^{*}\right\|$. Then $\left\{y_{n}\right\}_{n \in N}$ also forms a basis isomorphic to the basis $\left\{x_{n}\right\}_{n \in N}$ in $X$.

\section{Basic results}

Before giving the basic results we will prove the following auxiliary theorem.

Theorem 2 Let $p \in H^{\ln }$ and $p^{-}>1$. If the system

$$
\left\{e^{i(n-\alpha \operatorname{sign} n) t}\right\}_{n \in Z}
$$


forms a basis in $L_{p_{t}} \equiv L_{p_{t}}(-\pi, \pi)$, then this system is isomorphic to the classical system of exponents $\left\{e^{i n t}\right\}_{n \in Z}$, where the isomorphism is given by

$$
S f=e^{-i \alpha t} \sum_{0}^{\infty}\left(f, e^{i n x}\right) e^{i n t}+e^{i \alpha t} \sum_{1}^{\infty}\left(f, e^{-i n x}\right) e^{-i n t},
$$

where

$$
(f, g)=\frac{1}{2 \pi} \int_{-\pi}^{\pi} f(t) \overline{g(t)} d t
$$

Proof Consider the operator (4). From the basicity of system $\left\{e^{i n t}\right\}_{n \in Z}$ in $L_{p_{t}}$ it follows that $S$ is a bounded operator on $L_{p_{t}}$ into itself. It is easy to see that $\operatorname{Ker} S=0$. Actually, let $S f=0$. From the basicity of the system (3) in $L_{p_{t}}$ and from (4) we obtain $\left(f, e^{i n x}\right)=0, \forall n \in Z$. Also, from the basicity of system $\left\{e^{i n t}\right\}_{n \in Z}$ in $L_{p_{t}}$ it follows that $f=0$. We show that for all $g \in L_{p_{t}}$, the equation $S f=g$ in $L_{p_{t}}$ is solved. Let us assume that

$$
f=\sum_{n \in Z} g_{n} e^{i n t}
$$

where $\left\{g_{n}\right\}_{n \in Z}$ are the biorthogonal coefficients of the function $g$ by the system (3).

It is clear that $f \in L_{p_{t}}$, and so

$$
\begin{aligned}
S f & =e^{-i \alpha t} \sum_{n=0}^{\infty}\left(f, e^{i n x}\right) e^{i n t}+e^{i \alpha t} \sum_{n=1}^{\infty}\left(f, e^{-i n x}\right) e^{-i n t} \\
& =e^{-i \alpha t} \sum_{n=0}^{\infty} g_{n} e^{i n t}+e^{i \alpha t} \sum_{n=1}^{\infty} g_{-n} e^{-i n t}=g
\end{aligned}
$$

as by the condition of the theorem, the system (3) forms a basis in $L_{p_{t}}$.

This means that for all $g \in L_{p_{t}}$ the equation $S f=g$ is solved in $L_{p_{t}}$. Then by the Banach theorem the operator $S$ has a bounded inverse. It is obvious that $S\left[e^{i n t}\right]=A(t) e^{i n t}, n \geq 0$, and $S\left[e^{-i n t}\right]=B(t) e^{-i n t}, n \geq 1$. This completes the proof.

Now we study some basis properties of the system (1). Firstly, we recall the following theorem.

Theorem 3 ([11]) Let $p \in H^{\ln }$ and $p^{-}>1$. If parameter $\alpha \in$ R satisfies the condition $-\frac{1}{2 p(\pi)}<$ $\alpha<\frac{1}{2 q(\pi)}$, then the system $\left\{e^{i \mu_{n} t}\right\}$ forms a basis in $L_{p_{t}}$.

Let the asymptotics (2) occur. Let us assume $\mu_{n}=n-\alpha \operatorname{sign} n$ and $\delta_{n}=\lambda_{n}-\mu_{n}, \forall n \in Z$. It is easy to see that the inequality

$$
\left|e^{i \lambda_{n} t}-e^{i \mu_{n} t}\right| \leq c|n|^{-\beta}, \quad \forall n \neq 0
$$

is fulfilled, where $c$ is some constant. Let us assume that the following inequalities are satisfied:

$$
-\frac{1}{2 p(\pi)}<\alpha<\frac{1}{2 q(\pi)}, \quad \beta>\frac{1}{\tilde{p}},
$$


where $\tilde{p}=\min \left\{p^{-} ; 2\right\}$. Then, from Theorem 3 , the system of exponents $\left\{e^{i \mu_{n} t}\right\}_{n \in Z}$ forms a basis in $L_{p_{t}}$. By Theorem 1, it is isomorphic to the classical system of exponents $\left\{e^{i n t}\right\}_{n \in Z}$ in $L_{p_{t}}$. Therefore the spaces of coefficients of the bases $\left\{e^{i \mu_{n} t}\right\}_{n \in Z}$ and $\left\{e^{i n t}\right\}_{n \in Z}$ coincide.

Let $T: L_{p_{t}} \rightarrow L_{p_{t}}$ be a natural automorphism

$$
T\left[e^{i \mu_{n} t}\right]=e^{i n t}, \quad \forall n \in Z
$$

For all $f \in L_{p_{t}}$, let $\left\{f_{n}\right\}_{n \in Z}$ be biorthogonal coefficients of $f$ by the system $\left\{e^{i \mu_{n} t}\right\}_{n \in Z}$, and let $g=T f$. Therefore, $\left\{f_{n}\right\}_{n \in Z}$ are the Fourier coefficients of the function $g$ by the system $\left\{e^{i n t}\right\}_{n \in Z}$. From (4) and (5), it directly follows that

$$
\sum_{n \in Z}\left\|e^{i \lambda_{n} t}-e^{i \mu_{n} t}\right\|_{p_{t}}^{\tilde{p}}<+\infty
$$

Consider the following expression:

$$
\sum_{n}\left(e^{i \lambda_{n} t}-e^{i \mu_{n} t}\right) f_{n}
$$

We have

$$
\begin{aligned}
\left\|\sum_{n \in Z}\left(e^{i \lambda_{n} t}-e^{i \mu_{n} t}\right) f_{n}\right\|_{p_{t}} & \leq \sum_{n \in Z}\left\|e^{i \lambda_{n} t}-e^{i \mu_{n} t}\right\|\left|f_{n}\right| \\
& \leq\left(\sum_{n}\left\|e^{i \lambda_{n} t}-e^{i \mu_{n} t}\right\|_{p_{t}}^{\tilde{p}}\right)^{1 / \tilde{p}}\left(\sum_{n}\left|f_{n}\right|^{\tilde{q}}\right)^{1 / \tilde{q}},
\end{aligned}
$$

where $\frac{1}{\tilde{p}}+\frac{1}{\tilde{q}}=1$. By the Hausdorff-Young theorem [16], we have

$$
\left(\sum_{n}\left|f_{n}\right|^{\tilde{q}}\right)^{1 / \tilde{q}} \leq m_{1}\|g\|_{\tilde{p}}
$$

where $m_{1}$ is some constant. From $\tilde{p} \leq p^{-}$and the continuous embedding $L_{p_{t}} \subset L_{\tilde{p}}$, it follows that, $\exists m_{2}>0$,

$$
\|g\|_{\tilde{p}} \leq m_{2}\|g\|_{p_{t}} \leq m_{2}\|T\|\|f\|_{p_{t}} .
$$

As a result, we obtain

$$
\left\|\sum_{n}\left(e^{i \lambda_{n} t}-e^{i \mu_{n} t}\right) f_{n}\right\|_{p_{t}} \leq m_{1} m_{2}\|T\|\left(\sum_{n}\left\|e^{i \lambda_{n} t}-e^{i \mu_{n} t}\right\|_{p_{t}}^{\tilde{p}}\right)^{1 / \tilde{p}}\|f\|_{p_{t}} .
$$

Let us take $n_{0} \in N$ such that

$$
\delta=m_{1} m_{2}\|T\|\left(\sum_{|n|>n_{0}}\left\|e^{i \lambda_{n} t}-e^{i \mu_{n} t}\right\|_{p_{t}}^{\tilde{p}}\right)^{1 / \tilde{p}}<1 .
$$

Assume that

$$
\omega_{n}= \begin{cases}\lambda_{n}, & |n|>n_{0} \\ \mu_{n}, & |n| \leq n_{0}\end{cases}
$$


It is clear that the following inequality is satisfied:

$$
\left\|\sum_{n}\left(e^{i \omega_{n} t}-e^{i \mu_{n} t}\right) f_{n}\right\|_{p_{t}} \leq \delta\|f\|_{p_{t}} .
$$

It follows immediately from (7) that the expression $\sum_{n}\left(e^{i \omega_{n} t}-e^{i \mu_{n} t}\right) f_{n}$ represents a function from $L_{p_{t}}$ and it can be denoted by $T_{0} f$. Drawing attention to (8) we obtain $\left\|T_{0}\right\| \leq$ $\delta<1$. Thus, the operator $F=I+T_{0}$ is invertible, and it is easy to see that $F\left[e^{i \mu_{n} t}\right]=e^{i \omega_{n} t}$, $\forall n \in Z$. Hence, the system $\left\{e^{i \omega_{n} t}\right\}_{n \in Z}$ forms a basis in $L_{p_{t}}$ isomorphic to $\left\{e^{i \mu_{n} t}\right\}_{n \in Z}$. Systems $\left\{e^{i \lambda_{n} t}\right\}_{n \in Z}$ and $\left\{e^{i \omega_{n} t}\right\}_{n \in Z}$ differ in a finite number of elements. Therefore, by Statement 1 , the system $\left\{e^{i \lambda_{n} t}\right\}_{n \in Z}$ is complete in $L_{p_{t}}$, if $\lambda_{i} \neq \lambda_{j}$ for $i \neq j$. In the following it is necessary to apply Lemmas 1 and 2 .

As a result we obtain the following theorem.

Theorem 4 Let the asymptotics (2) occur and the inequalities

$$
-\frac{1}{2 p(\pi)}<\alpha<\frac{1}{2 q(\pi)}, \quad \beta>\frac{1}{\tilde{p}}
$$

be fulfilled, where $\tilde{p}=\min \left\{p^{-} ; 2\right\}$. Then the following properties of the system (1) are equivalent in $L_{p t}$ :

(1) the system (1) is complete;

(2) the system (1) is minimal;

(3) the system (1) is $\omega$-linear independent;

(4) the system (1) is isomorphic to $\left\{e^{i n t}\right\}_{n \in N}$ basis;

(5) $\lambda_{i} \neq \lambda_{j}$ for $i \neq j$.

Let us consider the case $\alpha=-\frac{1}{2 p(\pi)}$. In this case, by the results of [11], the system $\left\{e^{i \mu_{n} t}\right\}_{n \in Z}$ is complete and minimal in $L_{p_{t}}$, but it does not form a basis in it. Then from the previous considerations it follows that the system (1) cannot form a basis in $L_{p_{t}}$. Because otherwise, by Theorem 2, it will be isomorphic to system $\left\{e^{i n t}\right\}_{n \in Z}$ in $L_{p_{t}}$, and as a result the system $\left\{e^{i \mu_{n} t}\right\}_{n \in Z}$ should form a basis in $L_{p_{t}}$. This gives a contradiction.

By $\left\{v_{n}\right\}_{n \in Z} \subset L_{q_{t}}$ we denote the system biorthogonal to $\left\{e^{i \mu_{n} t}\right\}_{n \in Z}$. It is clear that using the estimates from [4], for $v_{n}, n \in Z$, we establish that the following relation is true:

$$
\gamma=\sup _{n}\left\|v_{n}\right\|_{q_{t}}<+\infty
$$

Let $\beta>1$. Then it is clear that the following inequality is satisfied:

$$
\sum_{n}\left\|e^{i \lambda_{n} t}-e^{i \mu_{n} t}\right\|_{p_{t}}<+\infty
$$

Similarly to the previous case, we can show that the operator

$$
\tilde{T} f=\sum_{n} v_{n}(f)\left(e^{i \lambda_{n} t}-e^{i \mu_{n} t}\right), \quad \forall f \in L_{p_{t}},
$$

is bounded in $L_{p_{t}}$. Introducing the new system $\left\{e^{i \omega_{n} t}\right\}_{n \in Z}$ in the same manner we establish the completeness of the system (1) in $L_{p_{t}}$, if $\lambda_{i} \neq \lambda_{j}$ for $i \neq j$. Minimality of the system (1) 
in $L_{p_{t}}$ follows from Lemma 3. Thus, if $\lambda_{i} \neq \lambda_{j}$ for $i \neq j$ and $\beta>1$, then the system (1) is complete and minimal in $L_{p_{t}}$ if the condition $-\frac{1}{2 p(\pi)} \leq \alpha<\frac{1}{2 q(\pi)}$ is satisfied.

Consider the case $\alpha \notin\left[-\frac{1}{2 p(\pi)}, \frac{1}{2 q(\pi)}\right)$. Let, for example, $\alpha \in\left[\frac{1}{2 q(\pi)}, \frac{1}{2 q(\pi)}+\frac{1}{2}\right)$. Multiplication of each member of the system (1) by $e^{i \frac{t}{2}}$ does not affect its basis properties in $L_{p_{t}}$. After appropriate transformations we obtain the system

$$
e^{i\left[\tilde{\alpha}+\tilde{\alpha}_{0}\right] t} \bigcup\left\{e^{i \tilde{\lambda}_{n} t}\right\}_{n \in Z}
$$

where $\tilde{\alpha}=\alpha-\frac{1}{2}$ and

$$
\tilde{\lambda}_{n}=n-\tilde{\alpha} \operatorname{sign} n+O\left(|n|^{-\beta}\right), \quad n \rightarrow \infty .
$$

Denote by $\tilde{\alpha}_{0}$ the member of $O\left(|n|^{-\beta}\right)$ in (2), corresponding to $n=0$. It is easy to see that condition $\lambda_{i} \neq \lambda_{j}$ is equivalent to $\tilde{\lambda}_{i} \neq \tilde{\lambda}_{j}$. It is clear that $-\frac{1}{2 p(\pi)} \leq \tilde{\alpha}<\frac{1}{2 q(\pi)}$. Then, by the previous results, the system $\left\{e^{i \tilde{\lambda}_{n} t}\right\}_{n \in Z}$ is complete and minimal in $L_{p_{t}}$, and therefore the system (10), and at the same time the system (1), is complete, but it is not minimal in $L_{p_{t}}$. Continuing this process we find that the system (1) is not complete, but it is minimal for $\alpha<-\frac{1}{2 p(\pi)}$; and the system (1) is complete, but it is not minimal in $L_{p t}$ for $\alpha \geq \frac{1}{2 q(\pi)}$. Thus, the following theorem is proved.

\section{Theorem 5 We have:}

(I) Let the asymptotics (2) occur and the inequalities (9) be fulfilled, where $\tilde{p}=\min \left\{p^{-} ; 2\right\}$. Then the following properties of the system (1) are equivalent in $L_{p_{t}}$ :

(1.1) the system (1) is complete;

(1.2) the system (1) is minimal;

(1.3) the system (1) is $\omega$-linear independent;

(1.4) the system (1) is isomorphic to $\left\{e^{i n t}\right\}_{n \in N}$ basis;

(1.5) $\lambda_{i} \neq \lambda_{j}$ for $i \neq j$.

(II) Let $\beta>1$ and $\alpha=-\frac{1}{2 p(\pi)}$. Then the following properties of the system (1) in $L_{p_{t}}$ are equivalent:

(2.1) the system (1) is complete;

(2.2) the system (1) is minimal;

(2.3) $\lambda_{i} \neq \lambda_{j}$, for $i \neq j$.

Moreover, in this case the system (1) does not form a basis in $L_{p_{t}}$.

(III) Let $\beta>1$ and $\lambda_{i} \neq \lambda_{j}$, for $i \neq j$. Then the system (1) is complete and minimal in $L_{p_{t}}$ for $-\frac{1}{2 p(\pi)} \leq \alpha<\frac{1}{2 q(\pi)}$, and for $\alpha<-\frac{1}{2 \pi}$ it is not complete, but it is minimal; and for $\alpha \geq \frac{1}{2 q(\pi)}$ it is complete, but it is not minimal in $L_{p_{t}}$.

\section{Acknowledgements}

I wish to expresses my thanks to Prof. Bilal T Bilalov, Institute of Mathematics and Mechanics of National Academy of Sciences, Baku, Azerbaijan, for his kind help, careful reading, and making useful comments on the earlier version of the paper 


\section{References}

1. Paley, R, Wiener, N: Fourier Transforms in the Complex Domain. Am. Math. Soc., Providence (1934)

2. Young, RM: An Introduction to Nonharmonic Fourier Series. Academic Press, New York (1980)

3. Sedletskii, AM: Biorthogonal expansions in series of exponents on intervals of the real axis. Usp. Mat. Nauk 37(5(227)), 51-95 (1982)

4. Moiseev, El: Basicity of system of exponents, cosines and sines in L . Dokl. Akad. Nauk SSSR 275(4), 794-798 (1984)

5. Kováčik, O, Rákosník, J: On spaces $L^{p(x)}$ and $W^{k, p(x)}$. Czechoslov. Math. J. 41, 592-618 (1991)

6. Fan, $X$, Zhao, D: On the spaces $L^{p(x)}(\Omega)$ and $W^{m, p(x)}(\Omega)$. J. Math. Anal. Appl. 263, 424-446 (2001)

7. Kokilashvili, V, Samko, S: Singular integrals in weighted Lebesgue spaces with variable exponent. Georgian Math. J. 10(1), 145-156 (2003)

8. Sharapudinov, II: Topology of the space $\mathscr{L}^{p(t)}([0,1])$. Mat. Zametki 26(4), 613-632 (1979)

9. Sharapudinov, II: Some questions of approximation theory in $L^{p(x)}(E)$ spaces. Anal. Math. 33(2), 135-153 (2007)

10. Bilalov, BT, Guseynov, ZG: Bases from exponents in Lebesgue spaces of functions with variable summability exponent. Trans. Acad. Sci. Azerb. Ser. Phys.-Tech. Math. Sci. 27(1), 43-48 (2008)

11. Bilalov, BT, Guseynov, ZG: Basicity of a system of exponents with a piece-wise linear phase in variable spaces. Mediterr. J. Math. 9(3), 487-498 (2012)

12. Bilalov, BT, Guseynov, ZG: Basicity criterion for perturbed systems of exponents in Lebesgue spaces with variable summability. Dokl. Akad. Nauk, Ross. Akad. Nauk 436(5), 586-589 (2011)

13. Zinger, I: Bases in Banach Spaces. I. Springer, Berlin (1970)

14. Bilalov, BT: Bases of exponentials, cosines and sines formed by eigenfunctions of differential operators. Differ. Equ. 39(5), 652-657 (2003)

15. Levinson, N: Gap and Density Theorems. Collog. Publ., vol. 26. Am. Math. Soc., Providence (1940)

16. Zygmund, A: Trigonometric Series, vol. 1. Mir, Moscow (1965)

10.1186/1029-242X-2014-495

Cite this article as: Muradov: On bases from perturbed system of exponents in Lebesgue spaces with variable summability exponent. Journal of Inequalities and Applications 2014, 2014:495

\section{Submit your manuscript to a SpringerOpen ${ }^{\circ}$ journal and benefit from:}

- Convenient online submission

- Rigorous peer review

- Immediate publication on acceptance

Open access: articles freely available online

- High visibility within the field

- Retaining the copyright to your article 